\title{
Individualized Low-Amplitude Seizure Therapy: Minimizing Current for Electroconvulsive Therapy and Magnetic Seizure Therapy
}

\author{
Angel V Peterchev*, 1,2,3, Andrew D Krystal', Moacyr A Rosa ${ }^{4}$ and Sarah H Lisanby ${ }^{1,5}$ \\ 'Department of Psychiatry and Behavioral Sciences, Duke University, Durham, NC, USA; ${ }^{2}$ Department of Biomedical Engineering, Duke University, \\ Durham, NC, USA; ${ }^{3}$ Department of Electrical and Computer Engineering, Duke University, Durham, NC, USA; ${ }^{4}$ Institute for Advanced Research in \\ Neurostimulation, São Paulo, Brazil; 5 Department of Psychology and Neuroscience, Duke University, Durham, NC, USA
}

\begin{abstract}
Electroconvulsive therapy (ECT) at conventional current amplitudes (800-900 mA) is highly effective but carries the risk of cognitive side effects. Lowering and individualizing the current amplitude may reduce side effects by virtue of a less intense and more focal electric field exposure in the brain, but this aspect of ECT dosing is largely unexplored. Magnetic seizure therapy (MST) induces a weaker and more focal electric field than ECT; however, the pulse amplitude is not individualized and the minimum amplitude required to induce a seizure is unknown. We titrated the amplitude of long stimulus trains (500 pulses) as a means of determining the minimum current amplitude required to induce a seizure with ECT (bilateral, right unilateral, bifrontal, and frontomedial electrode placements) and MST (round coil on vertex) in nonhuman primates. Furthermore, we investigated a novel method of predicting this amplitude-titrated seizure threshold (ST) by a non-convulsive measurement of motor threshold (MT) using single pulses delivered through the ECT electrodes or MST coil. Average STs were substantially lower than conventional pulse amplitudes ( I 12-174 mA for ECT and 37.4\% of maximum device amplitude for MST). ST was more variable in ECT than in MST. MT explained 63\% of the ST variance and is hence the strongest known predictor of ST. These results indicate that seizures can be induced with less intense electric fields than conventional ECT that may be safer; efficacy and side effects should be evaluated in clinical studies. MT measurement could be a faster and safer alternative to empirical ST titration for ECT and MST.

Neuropsychopharmacology (20I5) 40, 2076-2084; doi:I0.I038/npp.20 I5.I22; published online 20 May 2015
\end{abstract}

\section{INTRODUCTION}

The characteristics of the electrical stimulus in electroconvulsive therapy (ECT), in conjunction with the seizure propagation and termination processes, are key determinants of the clinical outcome of ECT. Modifications in ECT technique, including briefer stimulus pulses, unilateral electrode placement, and dosing relative to the individual seizure threshold (ST), have improved the side effect profile of modern ECT without sacrificing its unparalleled efficacy. These modifications reduced the intensity and duration of the electrical stimulus and made it more focal (Lee et al, 2012; Peterchev et al, 2010b; Sackeim, 2004; Sackeim et al, 1994). Nevertheless, a significant proportion of patients receiving ECT still experience adverse effects, which impedes the broader application of this highly effective intervention (Goodman, 2011; Kellner et al, 2010; Semkovska and

* Correspondence: Dr AV Peterchev, Department of Psychiatry and Behavioral Sciences, Duke University Medical Center, Box 3620 DUMC, Durham, NC 27710, USA, Tel: + 919684 0383, Fax: + 919681 9962, E-mail: angel.peterchev@duke.edu

Received 23 December 2014; revised 31 March 2015; accepted 24 April 2015; accepted article preview online 28 April 2015
McLoughlin, 2010; Verwijk et al, 2012). Furthermore, despite the demonstrated utility of ST titration in improving the efficacy and tolerability of ECT, ST titration still has not been uniformly adopted. This is, in part, due to time and inconvenience, perceived risks of exposing patients to subconvulsive trains (Kim et al, 2007; Lyons and Symon, 2008; Tang and Ungvari, 2001), as well as the desire to not 'waste' a session with an ineffective threshold treatment. Thus, the field needs improved, practical means to individualize and deliver ECT safely.

Despite the fact that stimulus pulse amplitude is a major driver of the electric field intensity and focality in the brain, it has received surprisingly little attention in dosage optimization. At conventional pulse amplitudes, ECT induces an electric field strength that substantially exceeds the neural activation threshold in most brain structures (Deng et al, 2011; Lee et al, 2014), resulting in widespread, nonfocal stimulation. This is higher than necessary to induce seizures since ECT studies dating back to the 1940s as well as several small recent studies demonstrated the feasibility of low-amplitude currents inducing seizures (Liberson, 1953; Nahas et al, 2013; Peterchev et al, 2010b; Rosa et al, 2011, 2012). Furthermore, magnetic seizure therapy (MST) elicits 
generalized seizures with an induced electric field that is substantially less intense and more focal than conventional ECT (Deng et al, 2011, 2013; Lee et al, 2014). After a series of promising case reports and safety and feasibility assessments (Kayser et al, 2009; Kosel et al, 2003; Lisanby et al, 2003a, 2001), the efficacy and side effects of MST were evaluated in two larger studies of 13 patients (Fitzgerald et al, 2013) and 26 patients (Kayser et al, 2015). In these studies, the response rates were $38 \%$ and $69 \%$, and the remission rates were $15 \%$ and $46 \%$, respectively. A sub-component of the latter study compared MST with right unilateral (RUL) ECT at $3 \times$ ST in 20 patients, and found no significant differences in efficacy (Kayser et al, 2011). This study reported low cognitive side effects in both ECT and MST groups, with faster reorientation following MST. In addition to faster reorientation, Lisanby et al (2003a) found significantly superior acute cognitive performance after MST than ECT. Faster reorientation after MST compared with prior ECT treatments was reported as well (Kirov et al, 2008). Finally, Fitzgerald et al (2013) found no evidence of neurocognitive impairment with MST. Thus, lowering the ECT pulse amplitude may be a valuable means of improving safety further, but questions of optimal dosing remain.

Another drawback of conventional ECT administration is that the pulse amplitude is fixed for all patients. Even when ST is titrated and the dose is set relative to ST, this is done by adjusting the duration and frequency of the stimulus train but not the current amplitude, which does not compensate for the individual variation of the electric field strength in the brain (Peterchev et al, 2010b). Consequently, individual variation in anatomy results in variable strength and focality of the induced electric field, which could account for some of the differences in side effects and therapeutic efficacy across patients (Deng et al, 2014). Therefore, individualization of the pulse amplitude in ECT, as well as in MST, could help maintain a consistent electricity exposure of the brain to compensate for individual anatomical differences (Deng et al, 2013; Lee et al, 2013b).

One approach to individualize the pulse amplitude is by titrating ST with stimulus trains with increasing amplitude (Liberson, 1948, 1953; Nahas et al, 2013). This approach, however, has some of the disadvantages of conventional ST titration as it exposes the patients to multiple subthreshold trains and a seizure induction with a threshold stimulus that may be subtherapeutic. Addressing these limitations, we individualize the ECT and MST pulse amplitude by titrating the motor threshold (MT) with a sequence of single pulses delivered through the ECT electrodes or MST coil, respectively. This approach is analogous to MT titration to individualize transcranial magnetic stimulation (TMS; Rossini et al, 1994) and the use of transcranial electric stimulation (TES) for intraoperative monitoring under anesthesia (Macdonald et al, 2013). Since MT captures individual variability in anatomy and neurophysiology, as well as the effects of the anesthesia, we hypothesize that it predicts the pulse-amplitude-titrated ST in ECT and MST. This approach could provide a novel method of individualizing dose without the need to administer multiple subconvulsive stimuli and without the need for seizure induction at a subtherapeutic dosage.

In this study, we characterize amplitude-titrated seizure induction with both electric and magnetic stimulation in nonhuman primates (NHPs), and test whether MT is a strong predictor of ST titrated in the amplitude domain.

\section{MATERIALS AND METHODS}

This study was approved by the Institutional Animal Care and Use Committees of New York State Psychiatric Institute, Columbia University, and Duke University. We previously described in detail methods for NHP models of ECT and MST (Lisanby et al, 2003b; Moscrip et al, 2004). Additional methodological details are provided as Supplementary Information.

\section{Subjects}

The subjects were male Macaca mulatta. Eight subjects participated in the MST condition (age $=12.0 \pm 2.8$ years, range $=9.0-17.7$ ). A subset of seven of these subjects took part in the bilateral (BL) and RUL ECT conditions (age = $12.3 \pm 2.7$ years, range $=10.0-16.6)$, and four of those participated in the bifrontal (BF) and frontomedial (FM) ECT conditions (age $=13.3 \pm 2.7$ years, range $=11.2-17.8$ ). The subjects' weight ranged from 8.2 to $16 \mathrm{~kg}$. Before the initiation of this study all subjects had been receiving ECT and MST regularly as part of other studies and piloting.

\section{Design}

Five seizure induction conditions were studied in the following order: vertex-round-coil MST, BL ECT, RUL ECT, BF ECT, and FM ECT. These conditions approximated in NHP the corresponding clinical stimulation configurations. One condition was tested per experimental session consisting of MT titration followed by ST titration under anesthesia. Each condition was repeated three times per subject. For each subject, experimental sessions were separated by at least 5 days to minimize carryover effects that could affect MT and ST (Spellman et al, 2009). Across subjects and stimulation modalities, a total of 90 stimulation sessions are included in the study.

\section{Anesthesia and Monitoring}

Anesthesia and monitoring were as described previously (Spellman et al, 2009). Aside from the pre-intervention sedation, which is not needed with humans, the anesthesia was modeled after the general anesthesia used in clinical ECT and MST to reproduce the underlying physiological state during seizure induction. Briefly, pre-intervention sedation was achieved with ketamine $(5-10 \mathrm{mg} / \mathrm{kg}$ i.m.) and xylazine (0.35-0.7 mg/kg i.m.). Atropine (0.04 mg/kg i.v.) was administered to reduce seizure-induced secretions, protect the airway, and protect from bradycardia that can result from subconvulsive stimulation. Before seizure titration, methohexital ( $1 \mathrm{mg} / \mathrm{kg}$ i.v.) and succinylcholine $(3.5 \mathrm{mg} / \mathrm{kg}$ i.v.) were administered for anesthesia and muscle relaxation, respectively. Sedation and anesthesia doses were adjusted based on earlier response, and additional boluses were administered during the procedure as needed. Two channels of BL fronto-mastoid electroencephalography (EEG) and one channel electrocardiography were recorded using a MECTA Spectrum 5000Q ECT device (MECTA Corp., Tualatin, OR). 

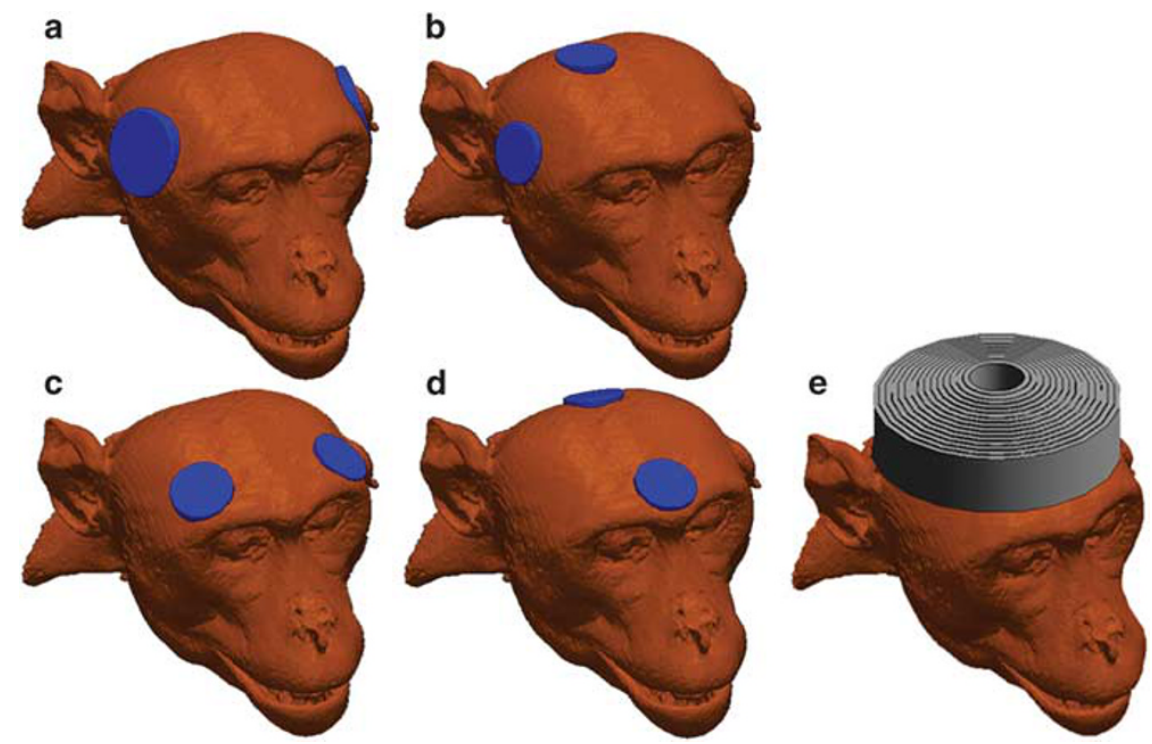

Figure I Illustration of ECT electrode and MST coil configurations used in the study. (a) Bilateral (BL) ECT, (b) right unilateral (RUL) ECT, (c) bifrontal (BF) ECT, (d) frontomedial (FM) ECT, (e) circular coil MST.

\section{Electric Stimulation}

Thymapad adhesive electrodes (Somatics, LLC, Lake Bluff, IL) were trimmed to $3.5 \mathrm{~cm}$ diameter circles for the $\mathrm{BL}$ configuration and to $2.5 \mathrm{~cm}$ circles for the RUL, BF, and FM configurations. The ECT electrode configurations are illustrated in Figure 1a-d. For the BL and BF configurations, the polarity of stimulation was defined as the polarity of the electrode contralateral to the hand for which the MT was determined. The polarity of RUL stimulation was defined as the polarity of the right temple electrode relative to the electrode near vertex. The polarity of FM stimulation was defined as the polarity of the posterior electrode relative to the anterior electrode.

A DS7AH constant-current stimulator (Digitimer, Welwyn Garden City, Hertfordshire, UK) was used for stimulus delivery for both the TES MT and ECT ST procedures. The pulse width was set to $0.2 \mathrm{~ms}$. To generate ECT stimulus trains, the DS7HA was triggered by an external function generator (Model \# 33521A, Agilent, Santa Clara, CA).

\section{Magnetic Stimulation}

A MagPro MST device with a $10-\mathrm{cm}$ diameter round coil (MagVenture A/S, Farum, Denmark) was used for both the TMS MT and MST ST procedures. The coil was centered at the vertex of the head, as illustrated in Figure 1e. The coil induced in the head a cosine current pulse with clockwise initial phase direction and a 0.36 -ms period. Stimulus intensity was reported as percentage of maximum pulse amplitude (\% MA).

\section{MT Titration}

MT was determined by delivering single stimulus pulses through the electrodes or coil used in the subsequent ECT or MST condition, respectively. Electromyography was measured from the first dorsal interosseous muscle in both hands for the BL, BF, and FM ECT and MST conditions, but only from the left hand in the RUL ECT condition, as the stimulation is unilateral in the right hemisphere. MT was defined as the minimum stimulus pulse amplitude needed to achieve $\geq 50-\mu \mathrm{V}$ peak-to-peak motor-evoked potential for at least 5 out of 10 trials (Rossini et al, 1994). MT was determined for both TES current polarities.

\section{ST Titration}

ST, reported in units of milliamperes $(\mathrm{mA})$, was determined by an ascending method of limits titration of the stimulus pulse amplitude. For the stimulation parameters used, ST can be converted to units of charge (mC) by multiplying the current amplitude by 0.1 . All stimulus parameters besides the pulse amplitude were held constant. The stimulus train consisted of 500 pulses delivered at 50 pulses per second resulting in train duration of $10 \mathrm{~s}$. Seizures were determined by observing the motor seizure manifestations in the cuffed left arm and EEG activity as a secondary criterion.

\section{Seizure Duration}

The motor seizure duration was determined based on observation of motor activity. The EEG seizure duration was determined by inspection of high-frequency polyspike activity in the EEG recording.

\section{Statistical Analysis}

The MT and ST data were first analyzed with mixed effects models to determine the effects of stimulation modality, session number, hand (for MT), polarity (for MT), and subject (random effect), as well as modality $\times$ session, modality $\times$ polarity, and hand $\times$ polarity interactions. Separate models were setup for ECT and MST as their measurement units of MT and ST are different and cannot 
be compared. The subject variance estimates from these analyses were used to calculate the coefficients of variation of MT and ST across subjects. Subsequently, we conducted a multiple regression predictor analysis of ST jointly for ECT and MST. To make the measurement units compatible between ECT and MST, MT and ST were normalized within each stimulation modality by calculating their z-scores, which capture the individual variability within modality. In addition to the normalized MT, the other evaluated predictors were session number, age, and weight as well as their interactions with stimulation modality. The predictors of motor and EEG seizure duration were analyzed similarly with a multiple regression with factors including stimulation modality as well as normalized ST, session number, age, weight and their interaction with stimulation modality. The seizure duration data were log-transformed to reduce the skewness of the distribution (Coffey et al, 1995). These multivariate analyses were followed up by Tukey's HSD test or Student's $t$-test to compare specific conditions. Further, we calculated a linear regression of the individual average ST on the individual average MT for each stimulation modality. In this analysis, we averaged MT across the hand and polarity conditions, and averaged MT and ST across sessions. In addition, we calculated the intraclass correlation coefficient (ICC) for MT prediction of ST (Fleiss, 1986; Streiner and Norman, 1995; Van Ness et al, 2008). The ICC represents agreement between the two evaluation methods-MT titration and ST titration. Using the ICC analysis, we compared ST with MT for each hand and polarity as well as the average MT across hand and polarity.

\section{RESULTS}

\section{Safety and Tolerability}

The procedures were well-tolerated and the subjects did not experience complications related to the procedures. The post-anesthesia recovery did not exceed $15 \mathrm{~min}$ after any session and no post-ictal agitation was observed. There were no cases of asystole or sustained bradycardia requiring intervention.

\section{Motor Threshold}

MT could be reliably determined in all subjects for all ECT electrode configurations and for MST. There was neither significant variation across the three sessions $(\mathrm{F}$ 's $<2.18$, $p$ 's $>0.142$ ) nor significant interaction between ECT modality and session $\left(\mathrm{F}_{3,202}=2.42, p=0.0673\right)$. MT did not differ significantly between the two hands ( $F$ 's $<3.09$, $p$ 's $>0.0806$; see Supplementary Table 1). MT was affected by ECT electrode configuration $\left(\mathrm{F}_{3,202}=8.44, p<0.0001\right)$ and current polarity $\left(\mathrm{F}_{1,202}=18.5, p<0.0001\right)$, as illustrated in Figure 2 . RUL had a higher MT than BL, BF, and FM by $25-35 \%$ $(p$ 's $<0.0001)$. BL, BF, and FM did not differ significantly in MT ( $p$ 's $>0.0523$ ). Anodal stimulation had lower MT than cathodal stimulation for all electrode configurations $(p<0.0001)$, with reduction ranging from $5.8 \%$ for FM to $14.6 \%$ for RUL. There were no significant interactions between polarity and either electrode configuration $\left(\mathrm{F}_{3,202}=\right.$ $1.02, p=0.387)$ or hand $\left(F_{1,202}=0.205, p=0.652\right)$.

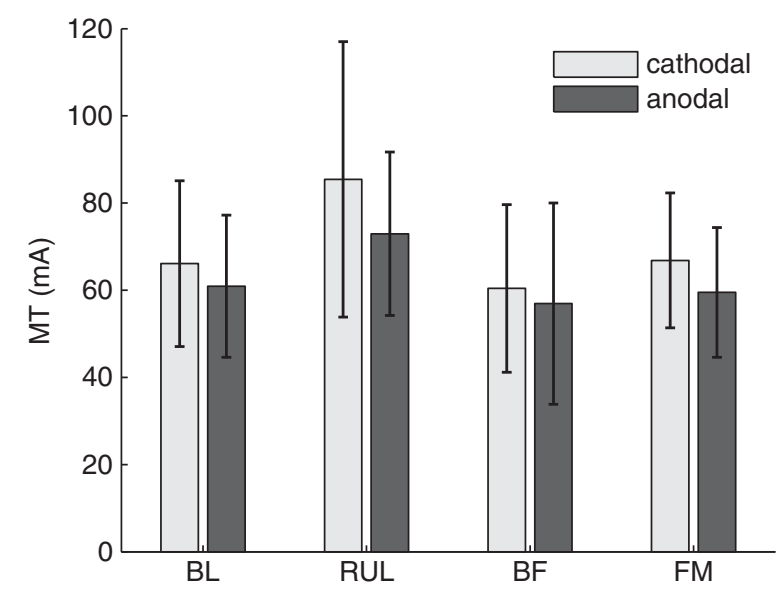

Figure 2 Comparison of motor threshold (MT) across ECT electrode configuration and current polarity. For the BL and BF configurations, polarity refers to the electrode contralateral to the hand for which MT was determined; for the RUL configuration, polarity refers to the temporal electrode relative to the vertex electrode; for the FM configuration, polarity refers to the posterior electrode relative to the anterior electrode. The bars show mean MT and the whiskers show SD. The MTs for the two hands did not differ significantly (see Supplementary Table I) and were averaged together. MT was affected significantly by both electrode configuration $(p<0.000 \mathrm{I})$ and current polarity $(p<0.000 \mathrm{I})$.

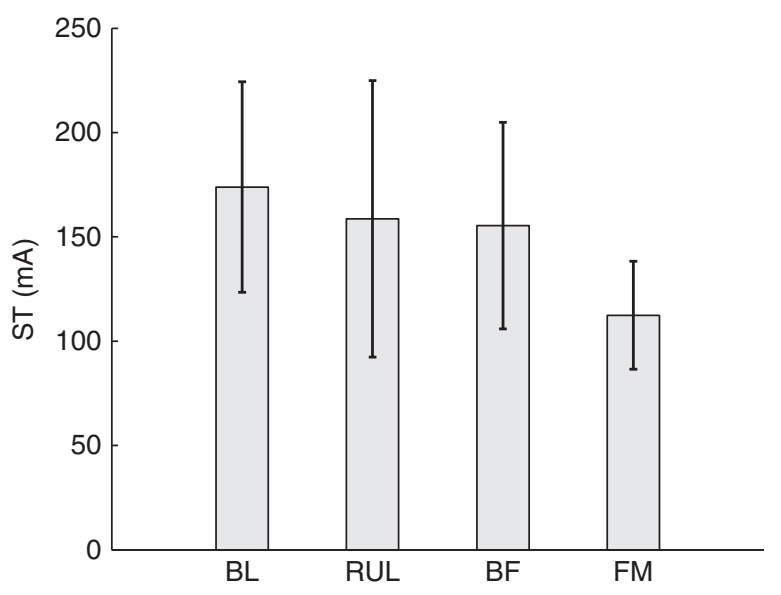

Figure 3 Seizure threshold $(S T$; mean $\pm S D$ ) for all ECT electrode configurations. The STs were significantly different between all ECT modalities ( $p$ 's $<0.0 I$ ), except between BL and RUL as well as between RUL and BF. ST can be converted to charge $(\mathrm{mC})$ by multiplying the value in $\mathrm{mA}$ by 0.1 .

\section{Seizure Threshold}

Titrating ST in the amplitude domain was feasible and resulted in seizures with both ECT and MST at substantially lower than conventional amplitudes. The average amplitudetitrated ST for MST was $37.4 \%$ MA with SD of $4.91 \%$ MA. The STs for the ECT modalities are shown in Figure 3. ECT electrode configuration affected ST significantly $\left(\mathrm{F}_{3,56.3}=\right.$ $20.9, p<0.0001)$. The average ECT STs ranged from $112 \mathrm{~mA}$ for $\mathrm{FM}$ to $174 \mathrm{~mA}$ for $\mathrm{BL}$, corresponding to 11.2 and $17.4 \mathrm{mC}$, respectively. The STs were significantly different 
between all ECT modalities ( $p$ 's $<0.01$ ), except between BL and RUL as well as between RUL and BF. For all ECT configurations and for MST, there was a significant variation across subjects of both ST and MT ( $p$ 's $<0.05)$. Notably, the variation of MT and ST among subjects was higher for electric stimulation than for magnetic stimulation, as illustrated in Figure 4.

The regression model indicated that within stimulation modality, ST was predicted significantly by only MT (averaged across hand and polarity; $\mathrm{F}_{1}=186, R^{2}=0.631$, $p<0.0001)$. In this model, ST was not predicted significantly by session, age, weight, or their interactions with stimulation modality ( $F$ 's $<3.29, p$ 's $>0.065$; for parameter estimates see Supplementary Table 2). Figure 5 shows the correlation between individual ST and MT within stimulation modality. $\mathrm{ST}$ and MT were averaged across sessions for each subject as

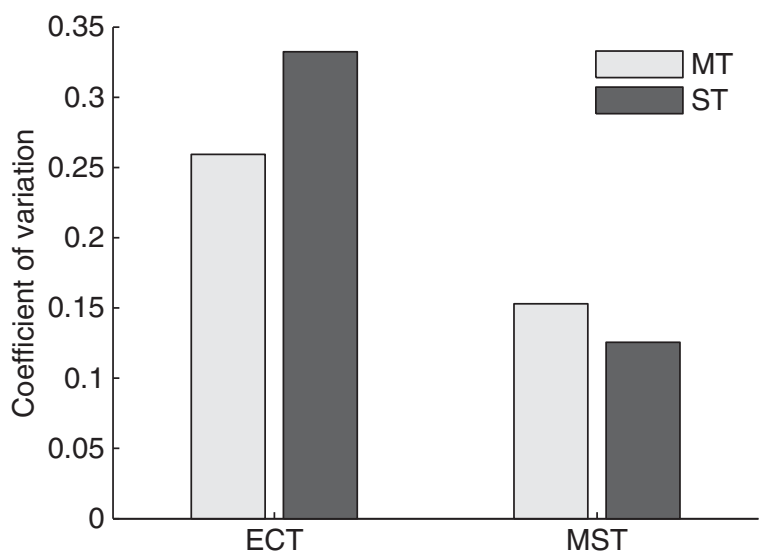

Figure 4 Coefficient of variation of MT and ST among the subjects for ECT and MST. session did not have a significant effect. The MT and ST were strongly correlated for all stimulation paradigms $\left(R^{2}\right.$ s $s>0.80$, $p$ 's $<0.02)$, except for BF ECT $\left(R^{2}=0.55, p=0.26\right)$. BF ECT showed a trend for ST increasing with MT, but the small number of subjects (four subjects) studied with this modality likely reduces the significance of this relationship. The ICCs comparing all of the available MT measures and ST were in the range of $0.60-0.84$, indicating moderate to strong agreement between the MT and ST measures (Fleiss, 1986; Streiner and Norman, 1995; Van Ness et al, 2008). The ICCs for the MTs averaged across hand and polarity are shown in Figure 5.

\section{Seizure Duration}

Representative seizure EEG tracings for one subject and the five stimulation modalities are shown in Supplementary Figure 1. Average motor and EEG seizure durations ranged from 17.4 and $16.5 \mathrm{~s}$ for MST to 23.7 and $22.3 \mathrm{~s}$ for BL ECT, respectively (see Supplementary Table 3 ). The only predictor of seizure duration that was significant for both motor and EEG seizures was age, which was associated with decreased duration $\left(F_{1}=15.9, p=0.0002\right.$; see Supplementary Table 4). In addition, the EEG seizure duration was inversely correlated with the individual ST $\left(\mathrm{F}_{1}=4.42, p=0.0394\right)$ and was significantly affected by the interaction between session and stimulation modality $\left(\mathrm{F}_{4}=2.90, p=0.0287\right)$.

\section{DISCUSSION}

We demonstrated that seizures can be induced in NHPs with ECT and MST current amplitudes much lower than in previous studies, and demonstrated for the first time individual dose titration in the pulse amplitude domain for several ECT
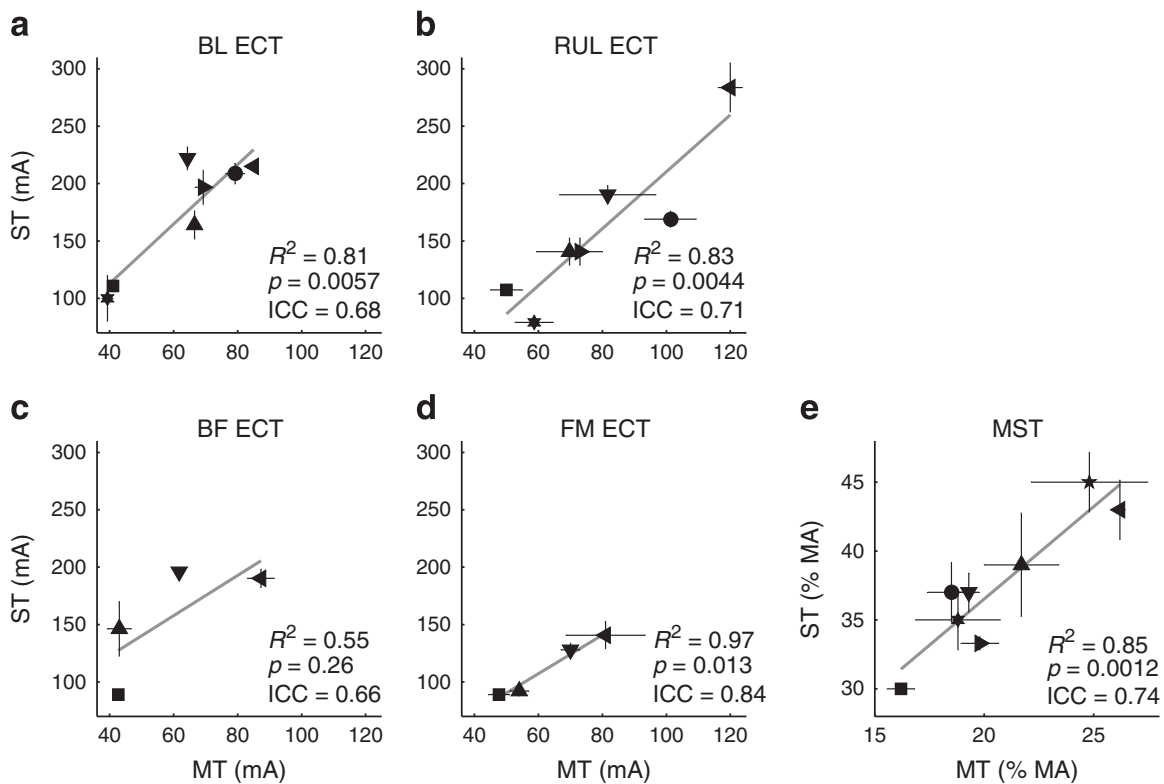

Figure 5 Correlation between individual MT and amplitude-titrated ST for BL, RUL, BF, and FM ECT (a-d) and MST (e). MT was averaged across hands and polarity. The symbols denote the mean value and error bars represent (population) SD across the three sessions. The ranges of the axes are matched for the ECT modalities $(a-d)$. The $R^{2}$ and $p$ values for linear regression as well as the intraclass correlation coefficient (ICC) are given for each configuration. In the combined analysis of all stimulation modalities, MT explained ST variance with $R^{2}=0.631$. 
electrode configurations as well as for MST under standard anesthesia. Furthermore, we introduced a novel approach to predicting ST using MT titration through the ECT electrodes or MST coil without having to induce a seizure. This new procedure for MT determination is the strongest known predictor of ST, explaining $63.1 \%$ of its variance in this study. The amplitude-titrated ST and MT both provide a reference for the minimum individual current amplitude, and hence the least intense and most focal stimulation, that can induce a seizure. This is significant because low-intensity, focal stimulation for seizure induction may be safer than conventional ECT paradigms that stimulate directly a large portion of the brain, as seen with MST.

\section{Stimulus Intensity}

In this study, seizures were induced with ECT current amplitudes that were only $12-22 \%$ of conventional values (800-900 mA). Although the ECT current amplitudes for NHPs are not directly comparable to those for humans, as human heads are of larger size and different anatomy than NHP heads, combining the NHP amplitude-titrated ST data with individual computational models of the electric field induced in the brain (Lee et al, 2013b) shows that the stimulation is substantially more focal than for human ECT with $800 \mathrm{~mA}$ (Deng et al, 2011; Lee et al, 2014). This suggests that the NHP STs would translate to currents lower than $800 \mathrm{~mA}$ in humans. Indeed, recent clinical studies have demonstrated seizure induction with currents as low as 400$500 \mathrm{~mA}$ (Nahas et al, 2013; Rosa et al, 2011, 2012). Notably, these clinical studies used stimulus trains with relatively few pulses ( $<160$ pulses). In contrast, we titrated ST with a train of 500 pulses, which could further reduce the current amplitude required for seizure induction (Liberson, 1948). Finally, the ECT pulse width in the NHP study $(0.20 \mathrm{~ms})$ was briefer than conventional ultrabrief ECT pules (0.25-0.30 $\mathrm{ms}$ ); wider pulses would be expected to have lower amplitude thresholds due to the strength-duration relationship (Liberson, 1945; Peterchev et al, 2010b). Thus, there is converging evidence that seizures can be induced with lower than conventional ECT current amplitude in humans as well. The resultant weaker and more focal electric fields are likely to reduce adverse side effects as evidenced by MST studies in NHPs (Moscrip et al, 2006; Spellman et al, 2008) and humans (see Introduction). However, the degree to which the current should exceed the amplitude-titrated ST to have a robust therapeutic effect, and the optimal selection of the other stimulus parameters, are unknown at present.

Although this study did not include stimulation at conventional high, fixed current strength, the range of average total charge (11.2 $\mathrm{mC}$ for FM to $17.4 \mathrm{mC}$ for $\mathrm{BL})$ was similar to that found in NHP studies of ECT with $800 \mathrm{~mA}$, $0.5 \mathrm{~ms}$ pulses for $\mathrm{BL}(17-18 \mathrm{mC})$, and FEAST $(14-18 \mathrm{mC})$ electrode configurations (Spellman et al, 2009), and somewhat higher than BL ECT with $800 \mathrm{~mA}, 0.15 \mathrm{~ms}$ pulses ( $8 \mathrm{mC}$; Lisanby et al, 2003b). Therefore, while in our study the current amplitude was more than fivefold lower, there was no apparent reduction in the total charge compared with studies with $800 \mathrm{~mA}$. This observation is corroborated by clinical studies of ultrabrief $(0.3 \mathrm{~ms})$ RUL ECT, where the ST charge for $800 \mathrm{~mA}$ current $(22 \pm 8 \mathrm{mC}$; Sackeim et al, 2008) was similar to that for $500 \mathrm{~mA}$ current $(21.6 \pm 4.8 \mathrm{mC}$; Rosa et al, 2011). In fact, for some stimulus parameter ranges, the total charge may increase with decreasing current amplitude (Swartz et al, 2012). The similarity in ST charge among modalities with very different strength and focality of stimulation by virtue of the different stimulus current amplitude supports the view that charge does not capture important dosing tradeoffs in ECT (Peterchev et al, 2010b).

We observed some significant differences in the amplitude-titrated STs across ECT electrode configurations. Therefore, the optimal current amplitude for various ECT electrode configurations may be different, whereas in present clinical practice the same current amplitude is used with all electrode placements. The FM electrode configuration had the lowest ST, potentially due to low-resistance current paths into the cranium through the orbits. This observation may be important for other ECT electrode configurations with anterior-posterior injected current flow such as FEAST (Nahas et al, 2013; Spellman et al, 2009). The observed ST variation may be confounded, however, by the differences in train directionality between electrode placements in this study (unidirectional with BL and RUL, and bidirectional with BF and FM) as well as by order effects associated with the sequence of stimulation modality conditions.

Finally, there was no significant effect of session number on the ST. This is in contrast to clinical ECT where ST increases with session number (Sackeim, 2004; van Waarde et al, 2013c). The most likely explanation for this difference is that our subjects had been receiving ECT and MST regularly before the initiation of this study and, within the study, the different stimulation modality conditions were scheduled without a significant break in between. Therefore, the subjects were effectively near a steady state with respect to seizure induction.

\section{Interindividual Threshold Variation and Stimulus Individualization}

In this study, we demonstrated that titrating ECT and MST ST by pulse amplitude adjustment, while keeping all other stimulus parameters fixed, reveals significant interindividual variability of the pulse amplitude required to induce a seizure. This observation is consistent with the understanding that individual anatomical differences (eg, head shape and size, scalp and skull thickness and conductivity) affect the electric field strength in the brain (Deng et al, 2014; Edwards et al, 2013; van Waarde et al, 2013a,b), and that pulse amplitude individualization can normalize it across individuals, as the electric field strength is directly proportional to the pulse amplitude (Deng et al, 2013; Peterchev et al, 2012).

We demonstrated that MT can be titrated with the same electrode and coil configuration used for seizure induction. The ability of this MT to explain $63.1 \%$ of the ST variance in our study is notable as known predictors of conventionally titrated ST for a given electrode placement explain less than $40 \%$ of the variance (Boylan et al, 2000; Chung, 2006; Galvez et al, 2015; Sackeim et al, 1994; van Waarde et al, 2013c). The strong correlation between MT and amplitude-titrated ST supports the explanation that the interindividual ST variability is influenced by individual anatomical parameters that also affect the MT. This explanation is further supported by our individual electric field models of four subjects from 
this study (Lee et al, 2013a, b, 2015). Therefore, MT titration might be considered as an alternative to the ST titration procedure, which may be safer since it does not entail the administration of multiple convulsive or near-convulsive pulse trains.

After accounting for electrode placement, age is the strongest predictor of conventionally titrated ST, although it explains only $10-30 \%$ of the ST variance (Boylan et al, 2000; Chung, 2006; Galvez et al, 2015; van Waarde et al, 2013c). Amplitude-titrated ST in unanesthetized patients also increases with age (Liberson, 1948). We did not find, however, a significant relation between age and ST. This is likely because the age range within each stimulation modality in this study was too truncated to detect age effects. Our analysis did not reveal weight as a significant predictor of ST either. This is largely consistent with clinical ECT data (Boylan et al, 2000; Chung, 2006; van Waarde et al, 2013c), except for a small predictive power of body mass index on BL ECT ST in one study (Chung, 2006).

The MT titration procedure in this study took a few minutes and was under a different type of anesthesia than the seizure induction. For the practical adoption of MT determination during an ECT or MST procedure, the MT titration should ideally be fast (no longer than a minute) and under the same anesthetic used for the seizure induction, as the patients are anesthetized for a relatively short period of time. This could be accomplished by using more efficient thresholding algorithms (Awiszus, 2003; Götz et al, 2011; Qi et al, 2011) and making the motor-evoked potentials under anesthesia more stable by delivering rapid bursts of 3-5 pulses instead of single pulses during the titration (Macdonald et al, 2013; Szelenyi et al, 2007).

Finally, the interindividual variability in both MT and ST was larger for ECT than for MST. This observation is consistent with our computational modeling studies and is likely explained by the higher sensitivity to most head anatomical parameters of the electric field induced in the brain by ECT compared with MST (Deng et al, 2014).

\section{Seizure Duration}

We observed inverse dependencies of motor and EEG seizure duration on age, as well as of EEG seizure duration on individual ST, consistent with clinical ECT with conventional dosing strategies (Boylan et al, 2000; Chung, 2002; Coffey et al, 1995; Rasimas et al, 2007). The reported significant interaction effect of session and stimulation modality on EEG seizure duration may be due to particularly long seizures occurring in arbitrary sessions that are different across the modalities. The lack of effect of weight on seizure duration is consistent with the literature especially when ST is added as a predictor (Boylan et al, 2000; Chung, 2002). Finally, we did not find significant differences in seizure duration across the stimulation modalities. This is consistent with our previous NHP studies of ECT and MST at conventional fixed, high-pulse amplitude (Cycowicz et al, 2008, 2009; Spellman et al, 2009). The average seizure durations for threshold and moderately suprathreshold trains in those studies (20-23s for ECT and 14-24s for MST) are consistent with our results as well. Therefore, on average, the seizure duration does not appear to have been affected substantially by the lower stimulus pulse amplitude.

\section{CONCLUSIONS}

We presented methods to determine the minimum current required to induce a seizure with various ECT and MST configurations. These include ST determination by pulse amplitude titration with a relatively long pulse train as well as a novel method of MT titration, which does not waste a treatment session and may be safer as it does not induce a seizure. Seizure induction with minimum current amplitude minimizes the electric field strength and maximizes its focality for a given electrode or coil configuration and therefore could potentially reduce side effects. It is possible, however, that the current amplitude required for robust therapeutic effect exceeds the amplitude-titrated ST. In a future clinical paradigm, the treatment stimulus amplitude could be set relative to the amplitude-titrated ST or MT based on a scaling factor that, together with the other pulse train parameters, has to be optimized in studies considering both tolerability and efficacy in humans. This work motivates further study of amplitude adjustment as a means of optimizing dosing in clinical ECT and MST.

\section{FUNDING AND DISCLOSURE}

Angel V Peterchev is inventor on patents, patent applications, and invention disclosures on TMS, MST, and ECT technology assigned to Columbia University and Duke University, including techniques described in this paper; he has received research support, patent royalties, and travel support from Rogue Research for TMS technology licensed to them; he has also received hardware donations from Magstim and travel support from Tal Medical related to TMS technology. Andrew D Krystal is inventor on a Duke University patent on ECT technology licensed to MECTA Corp. He receives no royalties from this patent. He receives research support from NIH grants (R01DK087717, R01HL096492, R01MH091053, R21NS085818, HHS-N2712012-000006-I, R01MH095780, R01MH078961), Teva, Sunovion, Phillips-Respironics, Eisai, Astellas, Abbott, Brainsway, ANS St. Jude, Neosync, Novartis, Janssen. He serves as a Consultant to Abbott, Janssen, AstraZeneca, Teva, Eisai, Jazz, Merck, Neurocrine, Novartis, Pfizer, Respironics, Roche, Sunovion, Somaxon, and Transcept. Moacyr A Rosa has no disclosures. Sarah H Lisanby has served as Principal Investigator on industry-sponsored research grants to Duke (ANS/St. Jude Medical, NeoSync, Brainsway, Nexstim); equipment loans to Duke (Magstim, MagVenture); is co-inventor on a patent and patent applications on TMS technology; is supported by grants from NIH (R01MH091083, 5U01MH084241, 5R01MH060884), Stanley Medical Research Institute, Brain and Behavior Research Foundation, and the Wallace $\mathrm{H}$. Coulter Foundation; and has no consultancies, speakers bureau memberships, board affiliations, or equity holdings in related device industries.

\section{ACKNOWLEDGMENTS}

We thank Brian Chan, Niko Reyes, Mohamed Aly, Christopher Sikes-Keilp, and Nagy Youssef for assisting in the NHP procedures and data entry, Won Hee Lee and ZhiDe Deng for preparing Figure 1, Christopher Sikes-Keilp and 
Zhi-De Deng for EEG data processing, and Maragatha Kuchibhatla and Bruce Luber for data analysis advice. This study was supported by NIH grants R01MH091083 and R01MH060884 and with MST device loan from MagVenture. This work was presented, in part, in preliminary form at the annual meetings of the International Society for ECT and Neurostimulation in 2010 (Peterchev et al, 2010a), the American College of Neuropsychopharmacology in 2011 and 2013, and the Society of Biological Psychiatry in 2014.

\section{REFERENCES}

Awiszus F (2003). TMS and threshold hunting. In:Paulus W, Tergau F, Nitsche MA, Rothwell JC, Ziemann U, Hallett M (eds). Transcranial Magnetic Stimulation and Transcranial Direct Current Stimulation (Supplements to Clinical Neurophysiology). Elsevier Science: Amsterdam, The Netherlands, Vol 56, pp 13-23.

Boylan LS, Haskett RF, Mulsant BH, Greenberg RM, Prudic J, Spicknall K et al (2000). Determinants of seizure threshold in ECT: benzodiazepine use, anesthetic dosage, and other factors. J ECT 16: 3-18.

Chung KF (2002). Relationships between seizure duration and seizure threshold and stimulus dosage at electroconvulsive therapy: implications for electroconvulsive therapy practice. Psychiatry Clin Neurosci 56: 521-526.

Chung KF (2006). Determinants of seizure threshold of electroconvulsive therapy in Chinese. J ECT 22: 100-102.

Coffey CE, Lucke J, Weiner RD, Krystal AD, Aque M (1995). Seizure threshold in electroconvulsive therapy: I. Initial seizure threshold. Biol Psychiatry 37: 713-720.

Cycowicz YM, Luber B, Spellman T, Lisanby SH (2008). Differential neurophysiological effects of magnetic seizure therapy (MST) and electroconvulsive shock (ECS) in non-human primates. Clin EEG Neurosci 39: 144-149.

Cycowicz YM, Luber B, Spellman T, Lisanby SH (2009). Neurophysiological characterization of high-dose magnetic seizure therapy: comparisons with electroconvulsive shock and cognitive outcomes. J ECT 25: 157-164.

Deng Z-D, Lisanby S, Peterchev A (2014). Effect of anatomical variability on electric field characteristics of electroconvulsive therapy and magnetic seizure therapy: a parametric modeling study. IEEE Trans Neural Syst Rehabil Eng 23: 22-31.

Deng Z-D, Lisanby SH, Peterchev AV (2011). Electric field strength and focality in electroconvulsive therapy and magnetic seizure therapy: a finite element simulation study. J Neural Eng 8: 016007 (016013pp).

Deng ZD, Lisanby SH, Peterchev AV (2013). Controlling stimulation strength and focality in electroconvulsive therapy via current amplitude and electrode size and spacing: comparison with magnetic seizure therapy. J ECT 29: 325-335.

Edwards D, Cortes M, Datta A, Minhas P, Wassermann EM, Bikson M (2013). Physiological and modeling evidence for focal transcranial electrical brain stimulation in humans: a basis for high-definition tDCS. Neuroimage 74: 266-275.

Fitzgerald PB, Hoy KE, Herring SE, Clinton AM, Downey G, Daskalakis ZJ (2013). Pilot study of the clinical and cognitive effects of high-frequency magnetic seizure therapy in major depressive disorder. Depress Anxiety 30: 129-136.

Fleiss JL (1986). The Design and Analysis of Clinical Experiments. John Wiley \& Sons: New York.

Galvez V, Hadzi-Pavlovic D, Smith D, Loo CK (2015). Predictors of seizure threshold in right unilateral ultrabrief electroconvulsive therapy: role of concomitant medications and anaesthesia used. Brain Stimul pii: S1935-861X(14)00449-5. doi:10.1016/j. brs.2014.12.012 (e-pub ahead of print).
Goodman WK (2011). Electroconvulsive therapy in the spotlight. N Engl J Med 364: 1785-1787.

Götz S, Whiting PA, Peterchev AV (2011). Threshold estimation with transcranial magnetic stimulation: algorithm comparison. Clin Neurophysiol 122: S197.

Kayser S, Bewernick B, Axmacher N, Schlaepfer TE (2009). Magnetic seizure therapy of treatment-resistant depression in a patient with bipolar disorder. J ECT 25: 137-140.

Kayser S, Bewernick BH, Grubert C, Hadrysiewicz BL, Axmacher N, Schlaepfer TE (2011). Antidepressant effects, of magnetic seizure therapy and electroconvulsive therapy, in treatment-resistant depression. J Psychiatr Res 45: 569-576.

Kayser S, Bewernick BH, Matusch A, Hurlemann R, Soehle M, Schlaepfer TE (2015). Magnetic seizure therapy in treatmentresistant depression: clinical, neuropsychological and metabolic effects. Psychol Med 45: 1073-1092.

Kellner CH, Knapp R, Husain MM, Rasmussen K, Sampson S, Cullum $M$ et al (2010). Bifrontal, bitemporal and right unilateral electrode placement in ECT: randomised trial. Br J Psychiatry 196: 226-234.

Kim C, Yokozuka M, Sato C, Nakanishi K, Kitamura A, Sakamoto A (2007). Incessant non-sustained ventricular tachycardia after stimulus of electroconvulsive therapy with atropine premedication? Psychiatry Clin Neurosci 61: 564-567.

Kirov G, Ebmeier KP, Scott AI, Atkins M, Khalid N, Carrick L et al (2008). Quick recovery of orientation after magnetic seizure therapy for major depressive disorder. Br J Psychiatry 193: 152-155.

Kosel M, Frick C, Lisanby SH, Fisch HU, Schlaepfer TE (2003). Magnetic seizure therapy improves mood in refractory major depression. Neuropsychopharm 28: 2045-2048.

Lee WH, Deng ZD, Kim TS, Laine AF, Lisanby SH, Peterchev AV (2012). Regional electric field induced by electroconvulsive therapy in a realistic finite element head model: influence of white matter anisotropic conductivity. Neuroimage 59: 2110-2123.

Lee WH, Lisanby SH, Laine AF, Peterchev AV (2013a). Anatomical variability predicts individual differences in ECT seizure threshold: a combined preclinical and computational modeling study. J ECT 29: 150-151.

Lee WH, Lisanby SH, Laine AF, Peterchev AV (2013b). Electric field characteristics of electroconvulsive therapy with individualized current amplitude: A preclinical study. Conf Proc IEEE Eng Med Biol Soc 2013: 3082-3085.

Lee WH, Lisanby SH, Laine AF, Peterchev AV (2015). Electric field model of transcranial electric stimulation in nonhuman primates: Correspondence to individual motor threshold. IEEE Trans Biomed Eng (e-pub ahead of print 22 April 2015, doi:10.1109/ TBME.2015.2425406).

Lee WH, Lisanby SH, Laine AF, Peterchev AV (2014). Stimulation strength and focality of electroconvulsive therapy and magnetic seizure therapy in a realistic head model. Conf Proc IEEE Eng Med Biol Soc 2014: 410-413.

Liberson WT (1945). Time factors in electric convulsive therapy. Yale J Biol Med 17: 571-578.

Liberson WT (1948). Brief stimulus therapy-physiological and clinical observations. Am J Psychiatry 105: 28-39.

Liberson WT (1953). Current evaluation of electric convulsive therapy; correlation of the parameters of electric current with physiologic and psychologic changes. Res Publ Assoc Res Nerv Ment Dis 31: 199-231.

Lisanby SH, Luber B, Schlaepfer TE, Sackeim HA (2003a). Safety and feasibility of magnetic seizure therapy (MST) in major depression: Randomized within-subject comparison with electroconvulsive therapy. Neuropsychopharm 28: 1852-1865.

Lisanby SH, Moscrip TD, Morales O, Luber B, Schroeder C, Sackeim HA (2003b). Neurophysiological characterization of magnetic seizure therapy (MST) in non-human primates. Clin Neurophysiol Suppl 56: 81-99. 
Lisanby SH, Schlaepfer TE, Fisch HU, Sackeim HA (2001). Magnetic seizure therapy of major depression. Arch Gen Psychiatry 58: 303-305.

Lyons JE, Symon J (2008). Asystole during electroconvulsive therapy in an elderly woman treated concomitantly with venlafaxine. Aust NZJ Psychiatry 42: 255.

Macdonald DB, Skinner S, Shils J, Yingling C (2013). Intraoperative motor evoked potential monitoring-A position statement by the American Society of Neurophysiological Monitoring. Clin Neurophysiol 124: 2291-2316.

Moscrip T, Terrace HS, Sackeim HA, Lisanby SH (2004). A primate model of anterograde and retrograde amnesia produced by convulsive treatment. J ECT 20: 26-36.

Moscrip TD, Terrace HS, Sackeim HA, Lisanby SH (2006). Randomized controlled trial of the cognitive side-effects of magnetic seizure therapy (MST) and electroconvulsive shock (ECS). Int $J$ Neuropsychopharmacol 9: 1-11.

Nahas Z, Short B, Burns C, Archer M, Schmidt M, Prudic J et al (2013). A feasibility study of a new method for electrically producing seizures in man: focal electrically administered seizure therapy [FEAST]. Brain Stimul 6: 403-408.

Peterchev AV, Chan B, Lisanby SH (2010a). Pulse amplitude adjustment: a novel means of individualizing and predicting dosage requirements for electroconvulsive therapy and magnetic seizure therapy. J ECT 26: 154-155.

Peterchev AV, Rosa MA, Deng ZD, Prudic J, Lisanby SH (2010b). Electroconvulsive therapy stimulus parameters: rethinking dosage. J ECT 26: 159-174.

Peterchev AV, Wagner TA, Miranda PC, Nitsche MA, Paulus W, Lisanby SH et al (2012). Fundamentals of transcranial electric and magnetic stimulation dose: definition, selection, and reporting practices. Brain Stimul 5: 435-453.

Qi F, Wu AD, Schweighofer N (2011). Fast estimation of transcranial magnetic stimulation motor threshold. Brain Stimul 4: 50-57.

Rasimas JJ, Stevens SR, Rasmussen KG (2007). Seizure length in electroconvulsive therapy as a function of age, sex, and treatment number. J ECT 23: 14-16.

Rosa MA, Abdo GL, Lisanby SH, Peterchev AV (2011). Seizure induction with low-amplitude-current electroconvulsive therapy. J ECT 27: 179.

Rosa MA, Abdo GL, Rosa MO, Lisanby SH, Peterchev AV (2012). Fronto-medial electrode placement with low current amplitude: a case report. J ECT 28: 146.

Rossini PM, Barker AT, Berardelli A, Caramia MD, Caruso G, Cracco RQ et al (1994). Non-invasive electrical and magnetic stimulation of the brain, spinal cord and roots: basic principles and procedures for routine clinical application. Report of an IFCN committee. Electroencephalogr Clin Neurophysiol 91: 79-92.

Sackeim HA (2004). Convulsant and anticonvulsant properties of electroconvulsive therapy: toward a focal form of brain stimulation. Clin Neurosci Res 4: 39-57.
Sackeim HA, Long J, Luber B, Moeller JR, Prohovnik I, Devanand DP et al (1994). Physical properties and quantification of the ECT stimulus: I. Basic principles. Convuls Ther 10: 93-123.

Sackeim HA, Prudic J, Nobler MS, Fitzsimons L, Lisanby SH, Payne N et al (2008). Effects of pulse width and electrode placement on the efficacy and cognitive effects of electroconvulsive therapy. Brain Stimulat 1: 71-83.

Semkovska M, McLoughlin DM (2010). Objective cognitive performance associated with electroconvulsive therapy for depression: A systematic review and meta-analysis. Biological Psychiatry 68: 568-577.

Spellman T, McClintock SM, Terrace HS, Luber B, Husain MM, Lisanby SH (2008). Differential effects of high-dose magnetic seizure therapy and electroconvulsive shock on cognitive function. Biol Psychiatry 63: 1163-1170.

Spellman T, Peterchev AV, Lisanby SH (2009). Focal electrically administered seizure therapy: A novel form of ECT illustrates the roles of current directionality, polarity, and electrode configuration in seziure induction. Neuropsychopharm 34: 2002-2010.

Streiner DL, Norman GR (1995). Health Measurement Scales: A Practical Guide to their Development and Use, 2nd edn. Oxford University Press: New York.

Swartz CM, Krohmer R, Michael N (2012). ECT stimulus dose dependence on current separately from charge. Psychiatry Res 198: 164-165.

Szelenyi A, Kothbauer KF, Deletis V (2007). Transcranial electric stimulation for intraoperative motor evoked potential monitoring: Stimulation parameters and electrode montages. Clin Neurophysiol 118: 1586-1595.

Tang WK, Ungvari GS (2001). Asystole during electroconvulsive therapy: a case report. Aust NZJ Psychiatry 35: 382-385.

Van Ness PH, Towle VR, Juthani-Mehta M (2008). Testing measurement reliability in older populations: methods for informed discrimination in instrument selection and application. J Aging Health 20: 183-197.

van Waarde JA, van Oudheusden LJ, Heslinga OB, Verwey B, van der Mast RC, Giltay E (2013a). Patient, treatment, and anatomical predictors of outcome in electroconvulsive therapy: a prospective study. J ECT 29: 113-121.

van Waarde JA, van Oudheusden LJ, Tonino BA, van der Wee NJ, Verwey B, van der Mast RC et al (2013b). MRI characteristics predicting seizure threshold in patients undergoing electroconvulsive therapy: a prospective study. Brain Stimul 6: 607-614.

van Waarde JA, van Oudheusden LJ, Verwey B, Giltay EJ, van der Mast RC (2013c). Clinical predictors of seizure threshold in electroconvulsive therapy: a prospective study. Eur Arch Psychiatry Clin Neurosci 263: 167-175.

Verwijk E, Comijs HC, Kok RM, Spaans HP, Stek ML, Scherder EJ (2012). Neurocognitive effects after brief pulse and ultrabrief pulse unilateral electroconvulsive therapy for major depression: a review. J Affect Disord 140: 233-243.

Supplementary Information accompanies the paper on the Neuropsychopharmacology website (http://www.nature.com/npp) 